\title{
Erratum
}

\section{Laparoscopic-Assisted Versus Open Abdominoperineal Resection for Low Rectal Cancer: A Prospective Randomized Trial}

\author{
Simon S. M. Ng, FRCSEd (Gen), Ka Lau Leung, MD, FRCS (Edin), Janet F. Y. Lee, MD, \\ FRCSEd (Gen), Raymond Y. C. Yiu, FRCSEd (Gen), Jimmy C. M. Li, FRACS, Anthony Y. \\ B. Teoh, MRCS (Edin), and Wing Wa Leung, MSc
}

Department of Surgery, The Chinese University of Hong Kong, Prince of Wales Hospital, Shatin, New Territories, Hong Kong

Erratum to: $10.1245 / \mathrm{s} 10434-008-9895-0$

Annals of Surgical Oncology

In Table 3 of the article titled "LaparoscopicAssisted Versus Open Abdominoperineal Resection for Low Rectal Cancer: A Prospective Randomized Trial" by Ng et al (DOI: 10.1245/s10434-008-9895-0) the correct unit of measure for blood loss is $(\mathrm{mL}$, mean and range) not $(\mathrm{mL}$, mean $+/-\mathrm{SD})$ as published.

The correct table in its entirety is:

Published online October 24, 2008

The online version of the original article can be found under doi: 10.1245/s10434-008-9895-0

Address correspondence and reprint requests to: Simon S. M. Ng, FRCSEd (Gen); E-mail: simonng@surgery.cuhk.edu.hk Published by Springer Science+Business Media, LLC $\odot 2008$ The Society of Surgical Oncology, Inc.
TABLE 3. Perioperative outcomes

\begin{tabular}{llll}
\hline & Lap group & Open group & $P$-value \\
\hline $\begin{array}{l}\text { Operative time (minutes, } \\
\quad \text { mean } \pm \text { SD) }\end{array}$ & $213.5 \pm 46.2$ & $163.7 \pm 43.4$ & $<.001^{*}$ \\
$\begin{array}{l}\text { Blood loss (mL, mean } \\
\text { and range) }\end{array}$ & $321.7(0-3000)$ & $555.6(0-4720)$ & $.093^{\dagger}$ \\
$\begin{array}{l}\text { Postoperative analgesic } \\
\text { requirement (No. of } \\
\text { injections, mean and } \\
\text { range) }\end{array}$ & $6.0(0-47)$ & $11.4(0-49)$ & $.007^{\dagger}$ \\
$\begin{array}{l}\text { Visual analogue pain } \\
\text { score on postoperative } \\
\text { day 1 (mean } \pm \text { SD) }\end{array}$ & $4.5 \pm 2.1$ & $4.9 \pm 1.9$ & \\
$\begin{array}{l}\text { Time first passing flatus } \\
\text { (days, mean and range) }\end{array}$ & $3.1(1-22)$ & $4.6(1-14)$ & $<.01^{*}$ \\
$\begin{array}{l}\text { Time of first bowel } \\
\text { motion (days, mean } \\
\text { and range) }\end{array}$ & $4.3(1-22)$ & $6.3(3-14)$ & $<.001^{\dagger}$ \\
$\begin{array}{l}\text { Time to resume normal } \\
\text { diet (days, mean and } \\
\text { range) }\end{array}$ & $4.3(2-25)$ & $5.1(3-16)$ & $.001^{\dagger}$ \\
$\begin{array}{l}\text { Time to walk } \\
\text { independently (days, } \\
\text { mean and range) }\end{array}$ & $4.4(1-10)$ & $5.9(2-20)$ & $.005^{\dagger}$ \\
$\begin{array}{l}\text { Hospital stay (days, mean } \\
\text { and range) }\end{array}$ & $10.8(5-27)$ & $11.5(5-38)$ & $.55^{\dagger}$ \\
$\begin{array}{l}\text { Lymph nodes removed } \\
\text { (mean } \pm \text { SD) }\end{array}$ & $12.4 \pm 6.7$ & $13.0 \pm 7.0$ & $.72^{*}$ \\
$\begin{array}{l}\text { Direct cost (US\$, } \\
\text { mean } \pm \text { SD) }\end{array}$ & $9588 \pm 1683$ & $7517 \pm 1693$ & $<.001^{*}$ \\
\hline
\end{tabular}

$\mathrm{SD}$, standard deviation.

* $t$-test.

${ }^{\dagger}$ Mann-Whitney $U$ test. 